\title{
Probing Reynolds stress models of convection with numerical simulations: III. Compressibility modelling and dissipation
}

\author{
F. Kupka ${ }^{1}$ and H. J. Muthsam ${ }^{2}$ \\ ${ }^{1}$ Max-Planck-Institute for Astrophysics, Karl-Schwarzschild-Str. 1, 85741 Garching, Germany \\ email: fk@mpa-garching.mpg.de \\ ${ }^{2}$ Faculty of Mathematics, Univ. of Vienna, Nordbergstraße 15, A-1090 Vienna, Austria \\ email: Herbert.Muthsam@univie.ac.at
}

\begin{abstract}
We provide results from an extended 3D numerical simulation study of Reynolds stress models of stellar convection and probe the modelling of compressibility, pressure fluctuations, and dissipation of turbulent kinetic energy.
\end{abstract}

Keywords. Convection, turbulence, stars: interiors

\section{Introduction and motivation}

In Kupka \& Muthsam (2007a) we introduce our study of Reynolds stress models for turbulent convection and consider super-adiabatic temperature gradients and the convective (enthalpy) flux. The modelling of non-locality as represented by the third order moments (TOMs) of velocity and temperature fields is discussed in Kupka \& Muthsam (2007b). Here, we investigate the modelling of pressure fluctuations and compressibility. We also study the flux of the dissipation rate of turbulent kinetic energy. We focus on consistency tests in which second order moments and mean quantities are taken from the 3D numerical simulations. The latter have been perfomed with the ASCIC code (Muthsam et al. 1995, 1999), as in Kupka \& Muthsam (2007a,b). For a non-local Reynolds stress model an accurate approximation of pressure fluctuations and dissipation in the flow is as important as a proper model for the advection processes described by the TOMs. This can already be concluded from some of the results shown by Kupka \& Muthsam $(2007 \mathrm{a}, \mathrm{b})$ and we provide further evidence in this paper. In the following $w$ and $\theta$ denote the fluctuations of vertical velocity and temperature relative to their horizontal, ensemble averaged mean. Similarly, $p^{\prime}$ relates to pressure fluctuations around the ensemble average of the mean pressure. Finally, $\langle\epsilon w\rangle$ denotes the flux of the dissipation rate of turbulent kinetic energy $\epsilon$, which is required for the non-local (differential) equation of the dissipation rate that in turn allows avoiding the introduction of a mixing length into convection models (Canuto 1992, 1993).

\section{Fluxes of pressure fluctuations and dissipation rate}

We investigate the modelling of pressure fluctuations $p^{\prime}$ and the dissipation rate of turbulent kinetic energy $\epsilon$ by probing models for their fluxes. For the flux of the dissipation rate we consider two models: $\langle\epsilon w\rangle=c\left\langle q^{2} w\right\rangle / \tau=c\left\langle q^{2} w\right\rangle \epsilon /\left\langle q^{2}\right\rangle$ with $c \sim 0.6$ ( $q^{2}$ is the sum of the squares of all three components of velocity fluctuations: $u, v, w$ ) and a down-gradient model, $\langle\epsilon w\rangle=-k \mathrm{~d} \epsilon / \mathrm{d} z$ with $k$ proportional to turbulent viscosity $\nu_{\mathrm{t}}$ (and also turbulent conductivity $\chi_{\mathrm{t}}$ ). The former was used in Canuto (1992) with 

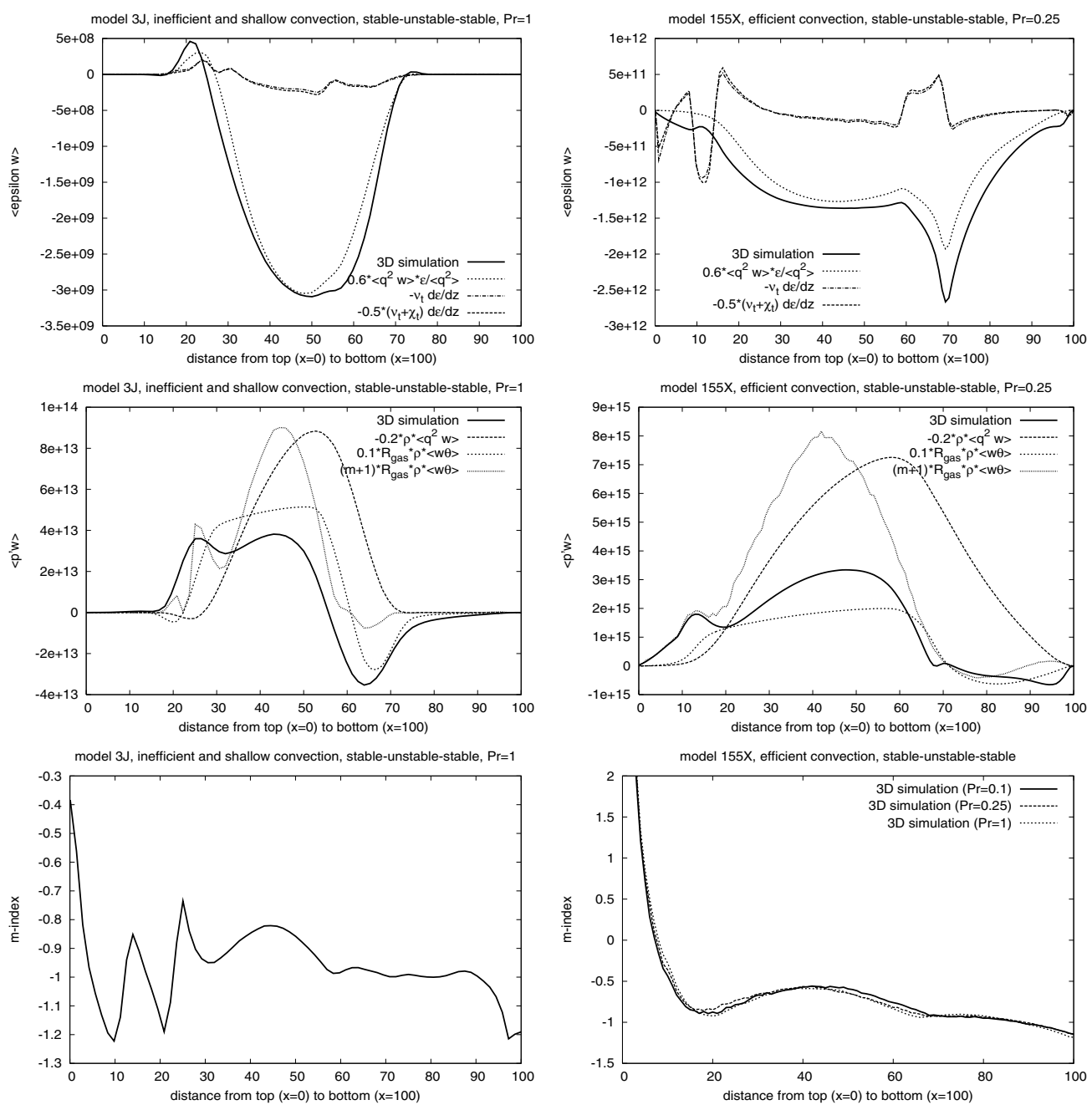

Figure 1. For cases ' $3 \mathrm{~J}$ ' (left column panels) and ' $155 \mathrm{X}$ ' (right column panels) consistency tests are shown for the non-local fluxes $\langle\epsilon w\rangle$ (top row) and $\left\langle p^{\prime} w\right\rangle$ (middle row). Input data for these models are taken from simulations and compared against direct computations. The bottom row shows the variation of the quantity $m$ with depth, as found in the numerical simulations. The dependence on Prandtl number Pr appears negligible. Values range from about -1 underneath to slightly above -1 inside convection zones with a more complex behaviour above them.

$c=1$ in the derivation of the TOM equations, the latter suggested in Canuto (1992) and Canuto \& Dubovikov (1998). For $\left\langle p^{\prime} w\right\rangle$ we consider three models: $\left\langle p^{\prime} w\right\rangle=-0.2 \rho\left\langle q^{2} w\right\rangle$, where $\rho$ is the mean density, as in Canuto $(1992,1993) ;\left\langle p^{\prime} w\right\rangle=(m+1) R_{\text {gas }} \rho\langle w \theta\rangle=$ $(m+1) P_{\text {gas }} / T\langle w \theta\rangle$, where the second variant assumes a perfect gas equation of state ( $R_{\text {gas }}$ is the gas constant, $P_{\text {gas }}$ the mean gas pressure, $T$ the mean temperature), suggested by Canuto (1997) (who required to weight averages with $\rho$, which provides only small corrections here and is thus neglected); and a variant thereof, where we take the polytropic index $n=1+1 / m$ as a constant. The index $n$ is used to model pressure fluctuations in Canuto (1997), not stellar structure or the equation of state of the gas. For $m=-1$, the Boussinesq approximation is recovered, while in general $m$ is obtained 
as the ensemble average of $\left(\rho^{\prime} / \rho\right)(\langle\rho T\rangle /(\langle\rho\rangle T-\langle\rho T\rangle))$. We computed the latter for our simulations ( $m$-index).

\section{Discussion of results and conclusions}

Although $\epsilon$ remains difficult to compute, an excellent scaling relation exists for its non-local flux $\langle\epsilon w\rangle$ provided a good model for the TOM $\left\langle q^{2} w\right\rangle$ is available, as we can see for both cases, '3J' and '155X', in Fig. 1 (see Kupka \& Muthsam 2007a for further details on these simulation runs). The down-gradient approximation for this quantity performs extremely poorly and should be avoided. It was also avoided in Kupka \& Montgomery (2002) and Montgomery \& Kupka (2004), who used the first model. For the pressure flux $\left\langle p^{\prime} w\right\rangle$ the available models are less convincing (Fig. 1). Clearly, the first (traditional) model, also quoted in Canuto $(1992,1993)$, provides just a crude estimate, which even yields the wrong sign in overshooting regions below the convection zone. The model suggested by Canuto (1997) provides a considerable improvement both in terms of accuracy and particularly in the ability to recover the correct direction of this flux. Evidently, the coupling between temperature and pressure field is important but ignored in the traditional model. However, the choice of $m$ remains difficult. From Fig. 1 we see it is everything but a constant and it certainly is not just -1 (the Boussinesq case), although this value is a first approximation for the overshooting region below convection zones. In similar regions above convection zones, large positive values are found, but they may be related to the upper, closed boundary conditions. We finally note that compressibility corrections are less important than the modelling of $\left\langle p^{\prime} w\right\rangle$ itself. Although we have not explicitly tested the models of Xiong $(1978,1986)$ and Xiong et al. (1997), we expect our conclusions to hold for them as well. Particularly, down-gradient approximations have to be considered with great caution, if more than integral quantities (such as radii) are to be predicted in detail.

\section{Acknowledgements}

H.J. Muthsam acknowledges support from FwF projects P17024 and P18224.

\section{References}

Canuto, V. M. 1992, ApJ 392, 218 (C'92)

Canuto, V. M. 1993, ApJ 416, 331 (C'93)

Canuto, V. M. 1997, ApJ 482, 827 (C'97)

Canuto, V. M. \& Dubovikov, M. S. 1998, ApJ 493, 834 (CD98)

Gryanik, V. M. \& Hartmann, J. 2002, J. Atmos. Sci. 59, 2729

Kupka, F. \& Montgomery, M. H. 2002, MNRAS 330, L6 (KM2002)

Kupka, F. \& Muthsam, H. J. 2007a, this volume p. 80

Kupka, F. \& Muthsam, H. J. 2007b, this volume p. 83

Montgomery, M. H. \& Kupka, F. 2004, MNRAS 350, 267

Muthsam, H. J., Göb, W., Kupka, F., Liebich, W. \& Zöchling, J. 1995, Astron. $\&$ Astrophys. 293,127

Muthsam, H. J., Göb, W., Kupka, F. \& Liebich, W. 1999, New Astronomy 4, 405

Xiong, D. R. 1978, Chinese Astronomy 2, 118

Xiong, D. R. 1986, Astron. \& Astrophys. 167, 239

Xiong, D. R., Cheng, Q. L. \& Deng, L. 1997, ApJS 108, 529 